\title{
Management of Salivary Hypofunction in Sjögren's Syndrome
}

\author{
Ava J. Wu, DDS \\ Address \\ 521 Parnassus Ave.; Rm C646, San Francisco, CA 94143, USA \\ Email: Ava.Wu@ucsf.edu
}

Published online: 8 August 2015

C) Springer International Publishing AG 2015

This article is part of the Topical Collection on Sjögren's Syndrome

Keywords Sjögren's syndrome $\cdot$ Dry mouth $\cdot$ Xerostomia $\cdot$ Pilocarpine $\cdot$ Cevimeline $\cdot$ Caries

\section{Opinion statement}

The strategies to increase oral comfort and manage dental decay can be time consuming and stressful for the patient with newly diagnosed Sjögren's syndrome who may have a host of other symptoms and regimens to manage. A conversation with the patient to assess their level of discomfort, the perceived time of most significant discomfort, and their perception of what would represent a successful outcome, combined with thorough clinical evaluation, can allow management of patient expectations and individualization of care. The goal is to keep the regimen simple. The vast majority of patients complaining of dry mouth use water as a wetting agent. If they complain of throat dryness or of water not being particularly effective, the addition of a small amount of oil (i.e., omega-3 or olive oil) to their water bottle may help the moisture adsorb to the mucosa of the oral cavity and throat. The artificial salivas and dry mouth products available on the market should ideally be of neutral $\mathrm{pH}$, the choice carefully made as to whether the product is beneficial, fits into their lifestyle, and is financially feasible. Self-stimulation of saliva via sugar-free chewing gum or hard candies may be implemented as needed throughout the day. The next level of care would be represented by stimulation of saliva with a parasympathomimetic medication. For some patients, we jump to this level of care immediately because it is the most effective for their lifestyle. We will often titrate the parasympathomimetic to the minimal effective dose, especially in petite females to allow them to take the medication without experiencing side effects. The expanded option of gargling with cevimeline/pilocarpine allows individuals to experience a local saliva-producing effects without potential systemic complications, but we do not yet have a good feel for how this is working in the clinical setting. Caries can best be prevented and the caries-repair process promoted with daily application of fluoride to the teeth (1.1\% NaF dentrifice available by prescription), daily removal of plaque from the tooth surfaces, and reducing acid production in dental plaque by controlling the intake of dietary sugar. For patients at high risk of caries, the same principles are applied with greater frequency, using higher concentration of fluoride and ancillary procedures as described in this paper. 


\section{Introduction}

This paper will focus on the clinical management of the salivary hypofunction in Sjögren's syndrome. Sjögren's syndrome (SS) is a chronic inflammatory autoimmune disease that prominently affects the salivary and lacrimal glands as well as other organ systems. Within the SS category, there is a spectrum of severity of the dry mouth complaint and of associated salivary gland dysfunction. The majority of individuals with SS will complain of significant dry mouth and have extremely low salivary production. A minority of individuals with SS will have a minor complaint of dry mouth and seemingly close to normal salivary gland function. Treatment may be individualized according to the severity of the complaint of dry mouth, the level of salivary dysfunction, assessment of caries risk, and the amount of time and effort that the patient is willing to expend on their oral care.

Specific clinical practice guidelines for the oral management of Sjögren's syndrome have been developed in concert with the Sjögren's Syndrome Foundation [1] and CAMBRA guidelines [2-4] may be used for the management of individuals at high risk for dental decay, which is the majority of SS patients. One will note that almost all of the practice guidelines for oral care are not supported by a strong scientific basis. The one exception is the use topical fluoride for the prevention of dental decay [1-5]. As a consequence, the management of salivary hypofunction in SS could be considered more art than science, but certainly the result of decades of experience.

The sensation of dry mouth occurs when the oral tissues do not smoothly glide. The cheeks and lips stick to the teeth, the tongue gets stuck to palate, food and epithelial debris collects in nooks and crannies, clicking of mucosa can be heard during speech, and swallowing can lead to choking. The oral tissue feels parched. The liquid film thickness on the palate, in particular, has been found to be decreased in those experiencing dry mouth.
Increasing comfort for these individuals is aimed at getting the tissues to glide smoothly. Saliva has lubricant properties and its volume may be increased in the oral cavity with medication to increase flow or alter the viscosity, sucking on sugar-free hard candies, chewing on sugar free gum or "gleeking" to self-express saliva during times of need. Alternatively, an artificial saliva or use of small sips of water with or without a small amount of oil (1/4 to 1 teaspoon of olive oil or omega-3 oil to $16 \mathrm{oz}$ of water, made fresh daily) may be used. The added oil aids in the movement of the oral soft tissues against the teeth and palate.

Saliva acts as an intraoral lubricant and has additional properties above and beyond providing intra-oral comfort. These functions include buffering, serving as a reservoir for molecules that can repair and protect the oral mucosa and dentition, providing volume to wash away detritus, and assisting with the act of swallowing and digestion. The loss of the ability to produce saliva is then thought to predispose to dental caries, oral infection, and difficulty with taste, swallowing, and buffering intraoral acid. The remainder of the treatment strategies for the oral component is aimed at duplicating the functions of saliva or defending against the effects of having reduced amounts of saliva.

The treatment of the oral component of SS is focused on several categories: increasing oral comfort, management of dental decay, management of oral soft tissue pathology, and stress and anxiety reduction. The treatment of the oral component of SS can be complex and time consuming for the patient and should tailored to their needs. It is beneficial if the practitioner can have a conversation with the patient with regard to when they are most dry, when they feel they want to have more saliva, and how much time they have to manage their oral dryness and potential sequelae.

\section{Treatment}

\section{Increasing salivary flow using medications}

The strategies to increase salivary flow in this patient population are most effective if there is residual salivary function. A quick evaluation of this may be done on an individual who has not had anything to eat or drink within the past $2 \mathrm{~h}$ (small sips of water are ok). If they are not able to expectorate any saliva into a container over a 5-min period, then it is likely their salivary function is diminished to the point where trying to stimulate saliva by 
pharmacologic means may not be helpful, but the patient may still consider a 12-week trial.

A muscarinic agonist (pilocarpine, cevimeline, bethanechol) is recommended to increase saliva production in patients with reduced salivary function. The rationale is that increasing the amount of saliva will result in all the positive benefits that saliva provides ( $\mathrm{pH}$ buffering, caries prevention, mucosal protection). The strength of this recommendation, however, is considered weak [1]. While the data are strong showing that muscarinic agonists increase saliva production, there are no studies showing that increasing saliva function results in decreased caries or oral infections. Stimulation of saliva with a muscarinic agonist such as pilocarpine, cevimeline, or bethanechol results in large amounts of saliva with low protein [6]. In contrast, stimulation of saliva via the sympathetic nervous system produces little saliva, but increased protein concentrate [6]. The implication is that the patient may produce a greater volume of saliva with the use of a muscarinic agonist, but it is unknown if they are actually getting more of the important, workhorse salivary constituents.

The available medications to stimulate salivary flow are dosed on a tid or qid regimen. The patient takes a pill and within $45 \mathrm{~min}$, they will note increased intraoral saliva. That increased salivary function will remain elevated for another 3 to $4 \mathrm{~h}$, with cevimeline having the longer duration (vs pilocarpine and bethanechol). As the salivary flow rate begins to fall after the effect of the muscarinic agonist wears off, the patient then takes another pill and the salivary flow rate is once again boosted for continuous salivary flow throughout the day. It is unknown if there are any long-term side effects from taking this medication.

Patients fall into several categories. They are either not interested in taking any systemic medication, leery of taking medication or interested in trying any medication. One also finds that these individuals may be further stratified into those who wish to have increased saliva all the time, those who only feel dry at night or upon awakening, and those who only need more saliva during meals. Thus, a patient may choose to only use medication at night or before a social/business occasion. Whatever regimen a patient chooses, I will usually have them try it for 12 weeks and then discontinue for a week to check for efficacy of the medication.

The dose of the muscarinic agonist may be titrated to the minimally effective dose to decrease the side effects (nausea, increased urination) while keeping the effect of increased salivation. With pilocarpine, the pill may be cut in half or quartered. The powder within the cevimeline capsule may be dissolved in water, but the final concentration is less precise.

The patient is informed that there are similar medications available to stimulate salivary flow and that they may need to try more than one for best results $[7,8 \bullet \bullet]$. Interestingly, ANA positivity was associated with failure of medication $[8 \bullet \bullet]$.

- Standard dosage: 5 or $7.5 \mathrm{mg}$ tid (max dose of $30 \mathrm{mg} /$ day)

- Contraindications: narrow angle glaucoma, asthma, acute iritis, gastric ulcer, use of $\beta$-blocker (may alter cardiac conduction). Caution should be used if the patient has asthma/COPD, chronic bronchitis, hepatic impairment, biliary disease, nephrothialiasis, and psychiatric disease 
- Main drug interactions: May antagonize other medications with anticholinergic effects (i.e., atropine, inhaled ipatromium, lomotil, diphenhydramine, promethazine, some phenothiazines, some antidepressants). Pilocarpine is a known inhibitor of CYP2A6 based on in vitro studies and may interact in vivo with coumadin

- Main side effects: sweating, rhinitis, increased urination

- Special points: Is a pill, so may be halved or quartered to determine the minimum effective dose. Ten milliliters of $1-2 \%$ solution of pilocarpine may be used as a 1-min gargle $[9,10]$

- Cost: $5 \mathrm{mg}$ (90 tablets), \$174; $7.5 \mathrm{mg}$ (100 tablets), \$247

\section{Cevimeline}

- Standard dosage: $30 \mathrm{mg}$ tid (maximum $90 \mathrm{mg} / \mathrm{day}$ )

- Contraindications: narrow angle glaucoma, acute iritis, asthma, $\beta$ adrenergic antagonist

- Main drug interactions: see pilocarpine above

- Main side effects: sweating, nausea, rhinitis, diarrhea, dyspepsia, and increased urination. May decrease visual acuity (especially at night) and depth perception

- Special points: $30 \mathrm{mg}$ of the powder may be dissolved in $5 \mathrm{ml}$ of water and used as a 2- to 4-min gargle to stimulate saliva production by the minor salivary glands [11]. The gargle is considered to not be as effective as taking cevimeline systemically [12]. The solution should contact areas with a high concentration of minor salivary glands-inner lip, buccal mucosa, floor of mouth, and especially the palate. The gargle may be used in those individuals where a systemic effect of the medication may be contraindicated

- Cost: $30 \mathrm{mg}$ (90 capsules), $\$ 288$

- Standard dosage: 10-25 mg PO tid-qid

- Contraindications: hyperthyroidism, peptic ulcer, latent or active bronchial asthma, pronounce bradycardia, or hypotension

- Main drug interactions: see pilocarpine above

- Main side effects: abdominal discomfort, salivation, flushing of the skin, sweating

- Special points: start at $10 \mathrm{mg}$ PO rid and titrate up or down depending on effect

- Cost: $10 \mathrm{mg}$ (30 each), $\$ 42$

Increasing saliva with non-pharmacologic methods

Masticatory or gustatory stimuli such as sugar-free gum or hard candies may be used to increase salivary flow to relieve oral symptoms. "Sugarless" products contain "less" sugar but remain cariogenic. Either method can provide an increase in salivary flow while the stimulus is in the mouth. The use of sugarfree 
chewing gum after meals has been associated with increasing the salivary film thickness, increasing clearance of food from the oral cavity, and balancing intraoral $\mathrm{pH}$ [13].

"Gleeking" is a method where saliva is expressed from submandibular/ sublingual glands by self-manipulation of the tongue against the palate or front teeth. This technique is useful in those who have observable salivary pooling and do not have water or other wetting agents available.

\section{Increasing oral comfort using wetting agents}

The use of saliva substitutes are helpful in allowing the oral tissues to glide over the teeth and offers temporary comfort. The choice of a specific product is subject to individual preference. So it is important that the patient be aware that finding the right product is a trial and error process [14]. The majority of these products only provide oral comfort; they do not prevent caries (unless fluoride is added) and do not treat intraoral fungal infections.

The saliva substitute should be applied thoroughly throughout the mouth with special attention to the hard palate $[13,15 \bullet \bullet]$ as the liquid film thickness in this area is reduced in those with dry mouth.

The shelf space for the American market is dominated by the Biotene product-line for dry mouth. The Biotene gel ( $\$ 5-8$ for $1.5 \mathrm{oz}$ ) is more viscous than the Biotene spray (contains castor oil \$6-9 for $1.5 \mathrm{oz}$ ). There are many other artificial salivas that have been developed for individuals who suffer from dry mouth, but are difficult to obtain and sample unless they go through a dental office, dental supply house, or internet.

Water is probably the most used wetting agent in this population. Most patients will carry a water bottle and may add a small bit of oil to their water bottle ( $1 / 4$ to $1 / 2$ teaspoon in $16 \mathrm{oz}$ ) to prolong the wetting effect. The oils most commonly used are olive oil or a liquid omega-3 oil purchased in the refrigerated section. Barlean's Omega Swirls ( $\$ 24$ for $16 \mathrm{oz}$ ) may be used to simultaneously add sugar-free flavor and oil to water. The patient should make their oil-water solution fresh daily and shake the bottle containing oil and water prior to sipping.

There is no strong evidence that any topical particular treatment is more effective in relieving the sensation of dry mouth [16]. The use of a topical wetting agent is a personal choice

\section{Dental caries prevention and treatment}

\section{Caries risk assessment}

In general, any individual with salivary hypofunction should be considered at high risk for developing caries $[2,3]$. Caries risk may be further evaluated by the presence of dry sticky mucosa (dental mirror or tongue depressor adheres to the buccal mucosa), visibly heavy plaque on the teeth, deep pits and fissures on the occlusal surface of the teeth, exposed root surface, new or recurrent caries on the areas that are considered highly cleansable (root surfaces, cusp tips, incisal edges), thick or no saliva expressed from the major salivary glands, the presence of erythematous candidiasis, glassy appearance to the oral mucosa, mucosal debris on palate, and orthodontic appliances. Other caries-promoting behaviors include frequent snacking ( $>3$ times daily between meals), recreational drug use, and the use of xerostomic medication $[2,3,17,18]$. 


\section{Diet}

Every patient should be educated on the role of dietary sugar in the promotion of caries. High risk patients should eliminate sugar (i.e., sucrose, glucose, and fructose) between meals. Between meal snacks that contain non-cariogenic sweetening agents (i.e., xylitol, sorbitol) are considered safer.

Xylitol is thought to have a role in caries prevention as it is not metabolized by cariogenic bacteria, leading to decreased acid production at the tooth surface and may contribute to a shift toward a less cariogenic bacterial population. There has been an explosion of xylitolcontaining gums, candies, and toothpastes on the market. Several guidelines $[2,3,19]$ recommend ingestion of 5-6 g of xylitol per day and/or the use of a xylitol-containing toothpaste. The evidence supporting these recommendations is considered weak [20•]

\section{Oral hygiene}

Effective plaque removal is critical to the caries prevention process. The patient should ask that their dental hygienist evaluate their brushing efficacy yearly by using a colored disclosing agent. The disclosing agents are now difficult for the individual consumer to obtain to self-assess their effectiveness. Patients will find that they will have specific areas that they will miss during brushing and those areas need to be identified.

Brushing twice daily with a soft toothbrush [21], the use of a fluoride-containing toothpaste, and daily use of dental floss are integral to effective oral hygiene. The most critical time to remove excess dental plaque is before going to sleep as the salivary flow rates fall very close to zero. Electric toothbrushes, irrigators, interdental toothpicks/brushes, dental wipes [22•], and supplementary dental aids are helpful.

\section{Fluoride}

Of all the recommendations for oral care, the use of fluoride has the most supportive evidence for its implementation $[1-5,23 \bullet]$. The Sjögren's Syndrome Foundation Guidelines for Oral Care have recommended that topical fluoride should be used in SS patients with dry mouth [1]. The strength of the recommendation was strong. Note that the recommendation is for individuals with "dry mouth" not "salivary dysfunction." The implication is that we believe that salivary flow has fallen $40-50 \%$ of baseline levels and some loss of protection from saliva has been lost.

Fluoride may be professionally applied or patient-applied. The SS patient with dry mouth should request a "neutral" fluoride be applied to their teeth at the dental office. The acidic product can strip the glaze from crown, composite fillings, and sealants, if present. In addition to using the 0.1 or $0.15 \%$ fluoride in OTC toothpaste twice daily, 
patients should receive supplemental forms of fluoride applied to the teeth.

\section{Professionally applied fluoride}

Acidulated phosphate fluoride (gel or foam)

- Standard dosage: $1.23 \%$

- Contraindications: hypersensitivity, mucositis, presence of crowns, composites, sealants (can create pitting)

- Main drug interactions: chlorhexidine (separate time of use by 12 to $24 \mathrm{~h}$ )

- Main side effects: allergy, local irritation, nausea, vomiting, and headache

- Special points: should be applied with caution in the SS population as the majority has crowns and composite fillings. Not to exceed four treatments per year. Dry-mouth patient may want to request that a "neutral" fluoride be applied in the dental office for greater comfort

- Cost: free to $\$ 50$ (varies by dental office)

Sodium fluoride varnish

- Standard dosage: $5 \%$

- Contraindications: ulcerative gingivitis, mucositis

- Main drug interactions: see above.

- Main side effects: edematous swelling, dyspnea, and nausea

- Special points: used for individuals at highest risk for dental caries and especially for those with exposed root surfaces [23•]. There is sustained release of the fluoride over time. The application of this product is done in the dental office and can be repeated ever 6 months or more frequently if necessary. Teeth may appear stained until brushed

- Cost: free to $\$ 50$ (varies by dental office, geographic location)

\section{Patient-applied fluoride}

- Standard dosage: $0.05 \%$

- Contraindications: hypersensitivity, mucositis

- Main side effects: hypersensitivity, nausea

- Special points: fluoride rinses in this category are alcohol free and likely to be more acceptable to those patients with sensitive mucosa. Rinse for 
1-2 min prior to bed. Used for those individuals who are at low to moderate risk of dental decay

- Cost: free to $\$ 50$ (varies by dental office, geographic location)

Neutral sodium fluoride gel or dentrifice

- Standard dosage: $1.1 \%$

- Contraindications: hypersensitivity, mucositis

- Main side effects: allergy, local irritation, nausea, vomiting, and headache

- Special points: the frequency of application can be daily to weekly depending on the frequency of recurrent caries. The fluoride is usually applied prior to bed. The patient will spit out any excess dentifrice so that the fluoride may sit on the teeth overnight. The gel may be used in a custom tray. The dental cream (Prevident 5000 Plus) may be brushed on and used instead of OTC $0.1 \% \mathrm{NaF}$ toothpaste. After application, of the fluoride with a tray or brush, floss or an interdental brush should be used to carry the fluoride to the interproximal spaces. Variations of the classic Prevident 5000 plus are available and usually need to be ordered: Prevident Dry Mouth where the foaming agent sodium lauryl sulfate has been removed and is good for individuals with sensitive mucosa, Prevident Sensitive/Enamel Protect that contains potassium nitrate to help with dental sensitivity to heat and cold, and Prevident Booster with the remineralizing agent tricalcium phosphate. There are multiple products in this category (ClinPro 5000 with calcium phosphate; X-Pur $1.1 \%$ NaF gel with xylitol)

- Cost: $\$ 25.00$ for 1 tube (51 g), $\$ 30$ for box of two tubes (102 g). A generic is available

\section{Root caries management}

The root surface of the tooth has a higher critical pH than enamel and is considered more susceptible to the caries process than enamel. The risk for caries on the root surface is especially pronounced when the gingiva recedes and exposes the root surface. Within the armamentarium of products to manage "root caries," there are a low number of clinical trials and a high risk of bias [24]. However, the data suggest that the root caries process can be slowed or even arrested. The two products most used in this category are a $5 \%$ fluoride varnish 2-4 times a year depending on risk [1$5]$ and the recently FDA-approved clorhexidine varnish (Cervitec, see below; [25]).

\section{Oral pH management}

The caries process and dental erosion are $\mathrm{pH}$-dependent processes. The buffering capacity is compromised in those individuals with the most severe salivary hypofunction. When a neutral or slightly basic $\mathrm{pH}$ cannot be maintained within 
mouth or on the tooth surface, the caries process can proceed unchecked. Acids may come from endogenous sources (gastric reflux, bacterial metabolism) and/or exogenous sources (carbonated beverages, sports drinks, juice, wine). To counter the effect of a sugary meal or an acidic challenge, the patient may simply rinse thoroughly with water, chew sugarfree gum immediately after, or brush their teeth to increase clearance of debris and plaque and to dilute the remaining intraoral acid. Intraoral buffering capacity may also be supplemented by the use of a bicarbonate mouth rinse (CariFree Maintenance rinse; homemade baking soda rinse with $1 / 2$ to 1 teaspoon in $16 \mathrm{oz}$ of water), toothpaste, soft chew (BasicBites $^{\circledR}$ ), or an extended release lozenge (Salese ${ }^{\circledR}$ ).

\section{Non-fluoride remineralizing agents}

These products deliver calcium and phosphate to the tooth surface facilitating restoration of demineralized areas [26]. They are not designed to replace fluoride, but rather to enhance its function. This approach can be effective, but only after the causes of demineralization are brought under control (i.e., poor oral hygiene, acid exposure). These agents are considered an adjunct therapy in patients with SS patients with dry mouth and a high root caries rate [1], as fluoride is considered the "gold standard" remineralizing agent $[27 \bullet \bullet]$.

1. Recaldent is casein phophopeptide (CPP) + amorphous calcium phosphate (APP). This product may be found in Trident Extra Care gum, MI Paste, and Meiji Milk Recaldent). MI paste is marketed with fluoride as MI Paste plus. MI paste has the advantage of having a smooth, slick feeling and is used secondarily as an oral lubricant.

2. Amorphous calcium phosphate. This product may be found in Arm and Hammer Age Defying Toothpaste, Discus ACP Oral Gel, and Premier Dental Enamel Pro Polishing Paste.

3. Sensitat which is arginine bicarbonate and calcium carbonate. This product may be found in Ortek Therapeutics' Denclude and Proclude line.

4. Novamin which is calcium sodium-phosphosilicate. The availability of this product varies from one country to another. In the USA, it is available as Nupro Sensodyne Prophy paste in the dental office. Sensodyne Repair and Protect toothpaste has Novamin in the Canadian/ UK version only.

5. Tricalcium phosphate that is marketed as ClinPro5000 toothpaste.

6. Nanohydroxyapatite that is marketed as Apagard ${ }^{\circledR}$ toothpaste.

7. Calcium glycerophosphate and sodium monofluorophophate that is marketed at Spry ${ }^{\circledR}$ toothpaste and oral rinse.

There is a dizzying array of interesting remineralizing agents on the market and coming to market. This is complicated by the fact that the various toothpaste recipes are periodically tweaked and re-formulated, so it can be difficult to track which toothpaste/rinse contains which product and how much of the product. In general, there is currently a lack of strong clinical data to support the use of 
these products $[1,28]$. There is the sense that these products are not harmful and may have the potential to help [29] and may be considered as adjunct therapy in SS patients with dry mouth [1].

\section{Antimicrobial agents for the prevention of caries}

Chlorhexidine administered by varnish, gel, or rinse may be considered in SS patients with dry mouth and root caries. The supporting evidence was deemed "weak" [1].

- Standard dosage: $0.12 \%, 15 \mathrm{ml}$ swish and spit bid for $30 \mathrm{~s}$

- Contraindications: hypersensitivity

- Adverse reactions: hypersensitivity/anaphylaxis, tooth staining (removable with dental cleaning), dental deposits, taste changes, local irritation

- Special points: used off label to decrease the amount of intraoral bacteria contributing to the caries process (use for 1 week every 3 months; CAMBRA) or to manage SS patients who have chronic, recurrent oral fungal infections. Peridex, PerioGard

- Cost: $0.12 \%$ (1 bottle of rinse, $473 \mathrm{ml}$ ), $\$ 18$

Chlorhexidine varnish

- Standard dosage: $1 \%$ chlorhexidine varnish +1\% thymol

- Contraindications: hypersensitivity

- Adverse reactions: mucous membrane irritation, reversible staining of the teeth

- Special points: Applied once every 3 months, more if the patient needs more intensive care. Approved by the FDA for treatment of root caries

- Cost: office charge

\section{Dental restoration}

In individuals with salivary hypofunction, the goal is to slow down or arrest the accelerated cycle of caries, restoration, restoration failure, and successive placement of a larger restoration until the ultimate failure resulting in extraction of the tooth. To slow down this cycle, only conservative intracoronal tooth restorations should be placed initially, with the goal of simply removing carious tooth structure and removing the minimum amount of tooth to preserve structural integrity [30]. Subgingival margins and full coronal coverage should be avoided wherever possible for initial treatment of these patients. This is because subgingival margins are the most common location of caries in individuals with salivary hypofunction. The subgingival margin is less accessible to topical fluoride and early caries is difficult to detect and restore in these areas. Full coverage crowns, if 
ultimately necessary, should not be placed until caries is under control (i.e., the patient has been free of new carious lesions for at least 1 year; interim restorations can be placed during the waiting period).

The major oral soft tissue problem for which SS patients require therapy is oral candidiasis that is almost invariably of the erythematous type. These individuals will typically complain of intraoral burning that is associated with the affected mucosa (tongue, palate, buccal mucosa, denture bearing mucosa). The intraoral lesions are frequently accompanied by cracking and redness at the corners of the lips-angular cheilitis, also of fungal origin requiring therapy.

If the patient wears a denture, it will need to be treated to prevent reinfection. If there is no metal associated with the denture, it may be soaked overnight in a dilute solution of 1:10 sodium hypochlorite:water and rinsed thoroughly prior to re-insertion. If the denture contains metal, it may be soaked twice daily in a chlorehexidine solution. The denturewearing patient may also sprinkle a very fine layer of nystatin or fluconazole powder onto the WET denture surface prior to insertion into the oral cavity.

The treatment end point occurs when the mucosal erythema has resolved, the filiform papillae of the dorsal tongue will re-emerge, and the associated burning sensation has resolved.

- Standard dosage: initial dose of $200 \mathrm{mg}$, then $100 \mathrm{mg} \mathrm{qd} \times 2$ weeks

- Contraindications: hypersensitivity

- Main drug interactions: inhibition of CYP2C9, CYP2C19, CYP3A4, UGT2B7, GI absorption enhanced by GLP-2 receptor antagonist

- Main side effects: nausea, vomiting, abdominal pain, diarrhea, dyspepsia, taste changes, dizziness, ALT/AST elevated

- Special points: convenient dosing. Patient should feel significant resolution of the oral burning within 3 to 4 days. If there is no resolution within one to two weeks consider switching to a topical medication, below. The fluconazaole may have been delivered in sub-therapeutic levels through the saliva

- Cost: $100 \mathrm{mg}$ (30 each), \$153

- Standard dosage: $10^{5}$ units/tablet qid $\times 10-14$ days

- Contraindications: hypersensitivity

- Main side effects: nausea/vomiting, diarrhea, abdominal pain

- Special points: intermittently available in the USA. The vaginal tablet is preferred for the SS population, as it contains no fermentable sugars. 
The product may be compounded as a sugar-free lollypop, pill, or liquid

- Cost: $\$ 80.00$ for 15 tablets

Miconazole mucoadhesive tablet

- Standard dosage: $50 \mathrm{mg}$ mucoadhesive tablet once daily $\times 14$ days

- Contraindications: Hypersensitivity, contains a milk protein, caution if hepatic impairment

- Main side effects: Anaphylaxis, neutropenia, diarrhea, headache, dysgeusia, upper abdominal pain, vomiting

- Drug interaction: Can enhance the effect of warfarin

- Special points: Sustained local release over several hours. Once daily application. Stays attached to mucosa for $6 \mathrm{~h}$ or more. No sugar in formulation. Cannot be used in patients with an upper denture interfering with placement of the drug in the canine fossa

- Cost: $\$ 270$ (14 tablets)

Clotrimazole troche

- Standard dosage: $10 \mathrm{mg}$ PO 5×/day $\times 14$ days

- Contraindications: hypersensitivity

- Main side effects: vomiting, nausea, abdominal cramps, abnormal LFT

- Special points: slowly dissolve in mouth, do not chew or swallow whole. Contains sugar. The individual with salivary hypofunction is advised to be scrupulous about oral hygiene, fluoride use, and diet during this period

- Cost: $10 \mathrm{mg}$ (70 each), \$129

- Standard dosage: $0.1 \%$ cream $/ 10^{5}$ units

- Contraindications: hypersensitivity

- Main side effects: burning, pruritis, irritation, dryness, folliculitis, hypertrichosis, allergic contact dermatitis, hypopigmentation, maceration, secondary infection, skin atrophy, striae, miliaria

- Special points: apply sparingly to corner of lips qid for 7-10 days

- Cost: $1 \mathrm{mg} / \mathrm{g} / 10^{5}$ units (1 tube of cream, $15 \mathrm{~g}$ ), \$96

\section{Other immune modulators and biologics}

Anti-TNF agents (infliximab, etanercept), dehydroepiandrosterone, glucocorticoids, anti-CD20 (rituximab), CTLA-4 fusion protein (abatacept), and plaquenil are currently not recommended for relieving the effects of salivary hypofunction associated with Sjögren's syndrome [6, 31, 32]. There is currently 
limited to no evidence showing that these drugs have any positive effect on salivary hypofunction in SS.

\section{Summary}

For management of salivary hypofunction in patients with SS, it is best to keep it simple. Finding the proper regimen to help with the sensation of dry mouth is a process of trial and error, but starts with simple measures like small sips of water throughout the day, learning to self-stimulate saliva using sugar-free hard candy/gum or the intraoral musculature, an artificial saliva at social occasions, or the use of a muscarinic agonist prior to bed or before a business meeting or eating. For the management of caries, a fluoride dentrifice (usually $1.1 \% \mathrm{NaF}$ available by prescription), meticulous oral hygiene, and control of sugar intake is preferred. Ancillary strategies may be added as the patient becomes proficient.

\section{Compliance with Ethics Guidelines}

\section{Conflict of Interest}

Ava J. Wu declares that she has no conflict of interest.

\section{Human and Animal Rights and Informed Consent}

This article does not contain any studies with human or animal subjects performed by the author.

\section{References and Recommended Reading}

Papers of particular interest, published recently, have been highlighted as:

- Of importance

- Of major importance

1. Zero D, Brennan M, Daniels T, Stewart C, Papas A, Pinto A, et al. Sjogren's syndrome foundation (SSF) clinical practice guidelines for oral disease management: caries prevention. Scand J Immunol. 2015;81:329-450.

2. Young DA, Featherstone JD. Caries management by risk assessment. Community Dent Oral Epidemiol. 2013;41(1):e53-63.

3. Featherstone JDB, Singh S, Curtis DA. Caries risk assessment and management for the prosthodontic patient. J Prosthodont. 2011;20(1):2-9.

4. Su N, Marek CL, Ching V, Miriam Grushka M. Caries prevention for patients with dry mouth. J Can Dent Assoc. 2011;77:b85.

5. Weyant RJ, Tracy SL, Anselmo TT, Beltrán-Aguilar ED, Donly KJ, Frese WA, et al. Topical fluoride for caries prevention: executive summary of the updated clinical recommendations and supporting systematic review. J Am Dent Assoc. 2013;144(11):1279-91. Erratum in: J Am Dent Assoc. 2013 Dec;144(12):1335. Dosage error in article text.

6. von Bültzingslöwen I, Sollecito TP, Fox PC, Daniels T, Jonsson R, Lockhart PB, et. al. Salivary dysfunction associated with systemic diseases: systematic review and clinical management recommendations. Oral Surg Oral Med Oral Pathol Oral Radiol Endod. 2007;103 Suppl:S57.e1-15.

7. Chainani-Wu N, Gorsky M, Mayer P, Bostrom A, Epstein JB, Silverman Jr S. Assessment of the use of sialogogues in the clinical management of patients with xerostomia. Spec Care Dentist. 2006;26(4):164-70. 
8.• Noaiseh G, Baker JF, Vivino FB. Comparison of the discontinuation rates and side-effect profiles of pilocarpine and cevimeline for xerostomia in primary Sjögren's syndrome. Clin Exp Rheumatol. 2014;32(4):575-7.

Reinforces the Chainani-Wu paper that there is currently a "trial and error" element to the selection of a muscarinic agonist to stimulate saliva

9. Bernardi R, Perin C, Becker FL, Ramos GZ, Gheno GZ, Lopes LR, et al. Effect of pilocarpine mouthwash on salivary flow. Braz J Med Biol Res. 2002;35(1):105-10.

10. Kim JH, Ahn HJ, Choi JH, Jung DW, Kwon JS. Effect of $0.1 \%$ pilocarpine mouthwash on xerostomia: doubleblind, randomised controlled trial. J Oral Rehabil. 2014;41(3):226-35.

11. Takagi Y, Katayama I, Tashiro S, Nakamura T. Parotid irrigation and cevimeline gargle for treatment of xerostomia in Sjögren's syndrome. J Rheumatol. 2008;35(11):2289-91.

12. Takagi Y, Kimura Y, Nakamura T. Cevimeline gargle for the treatment of xerostomia in patients with Sjögren's syndrome. Ann Rheum Dis. 2004;63(6):749. No abstract available.

13. Dibdin GH, Dawes C, Macpherson LM. Computer modeling of the effects of chewing sugar-free and sucrose-containing gums on the $\mathrm{pH}$ changes in dental plaque associated with a cariogenic challenge at different intra-oral sites. J Dent Res. 1995;74(8):1482-8.

14. United Kingdom Medicines Information. Saliva substitutes: choosing and prescribing the right product. 2013 Feb. www.evidence.nhs.uk

15.• Dawes C. How much saliva is enough for avoidance of xerostomia? Caries Res. 2004;38(3):236-40.

This is an older paper, but presents an interesting premise on how one can think about oral dryness in terms of increasing and maintaining the liquid film thickness in specific areas of the oral cavity.

16. Furness S, Worthington HV, Bryan G, Birchenough S, McMillan R. Interventions for the management of dry mouth: topical therapies. Cochrane Database Syst Rev. 2011;12:CD008934.

17. Navazesh M. How can oral health care providers determine if patients have dry mouth? J Am Dent Assoc. 2003;134(5):613-20.

18. Osailan S, Pramanik R, Shirodaria S, Challacombe SJ, Proctor GB. Investigating the relationship between hyposalivation and mucosal wetness. Oral Dis. 2011;17(1):109-14.

19. Council on Clinical Affairs. Guideline on xylitol use in caries prevention. Chicago (IL): American Academy of Pediatric Dentistry (AAPD). Adopted 2006, Revised 2010.

20. Riley P, Moore D, Ahmed F, Sharif MO, Worthington HV. Xylitol-containing products for preventing dental caries in children and adults. Cochrane Database Syst Rev. 2015;3:CD010743.

Concludes that the current evidence supporting the use of xylitol containing products for preventing dental caries due to scarcity and low quality of studies. This is otherwise an excellent review of the major studies behind the current use of xyitol in chewing gum and hard candies.

21. Zimmermann H, Zimmermann N, Hagenfeld D, Veile A, Kim TS, Becher H. Is frequency of tooth brushing a risk factor for periodontitis? A systematic review and meta-analysis. luCommunity Dent Oral Epidemiol. 2015;43(2):116-27.

22. Zhan L, Featherstone JD, Lo J, Krupansky C, Hoang N, DenBesten $P$, et al. Clinical efficacy and effects of xylitol wipes on bacterial virulence. Adv Dent Res. 2012;24(2):117-22.

A clever paper that emphasizes the role of using $2 \times 2$ guaze or xlitol containing wipe to physically remove plaque from the teeth can be an useful adjunct for plaque removal when a toothbrush is not available.

23. Richards D. Substantial reduction in caries from regular fluoride varnish application. Evid Based Dent. 2013;14(3):72-3.

Fluoride varnish is approved for caries management in children with a high caries rate. With the features of extended release and application of the fluoride directly to trouble areas, varnish is a useful tool in the armamentarium to manage adult SS patients at high risk for caries.

24. Hayes M. Topical agents for root caries prevention. Evid Based Dent. 2015;16:10-1.

25. Wierichs RJ, Meyer-Lueckel H. Systematic review on noninvasive treatment of root caries lesions. J Dent Res. 2015;94(2):261-71.

26. Spolsky VW, Black BP, Jenson L. Products-old, new, and emerging. J Calif Dent Assoc. 2007;35(10):72437.

27.• Zero DT. Dentifrices, mouthwashes, and remineralization/caries arrestment strategies. BMC Oral Health. 2006;6 Suppl 1:S9.

An excellent article on toothpastes and the rationale for the ingredients that they contain.

28. Amaechi BT, van Loveren C. Fluorides and nonfluoride remineralization systems. Monogr Oral Sci. 2013;23:15-26.

29. Rethman MP, Beltrán-Aguilar ED, Billings RJ, Hujoel PP, Katz BP, Milgrom P, et al. Nonfluoride cariespreventive agents: executive summary of evidencebased clinical recommendations. J Am Dent Assoc. 2011;14((9):1065-71.

30. Featherstone JD, Doméjean S. Minimal intervention dentistry: part 1. From 'compulsive' restorative dentistry to rational therapeutic strategies. Br Dent J. 2012;213(9):441-5.

31. Ramos-Casals M, Brito-Zerón P, Sisó-Almirall A, Bosch $\mathrm{X}$, Tzioufas AG. Topical and systemic medications for the treatment of primary Sjögren's syndrome. Nat Rev Rheumatol. 2012;8(7):399-411.

32. Gottenberg JE, Ravaud P, Puéchal X, Le Guern V, Sibilia $\mathrm{J}$, Goeb V, et al. Effects of hydroxychloroquine on symptomatic improvement in primary Sjögren's syndrome: the JOQUER randomized clinical trial. JAMA. 2014;312(3):249-58. 